\title{
Revisão sistemática sobre o Pole Dance
}

\author{
Systematic review on Pole Dance
}

Revisión sistemática sobre el Pole Dance

\section{Resumo}

O Pole Dance é um exercício físico que por sua vez utiliza do atrito e oposição entre o corpo do praticante e uma barra vertical para a realização de figuras e posturas plásticas, estáticas ou dinâmicas, com elementos da ginástica e acrobacias circenses, compondo danças ou sequências acrobáticas. Esta pesquisa sobre o Pole Dance teve como objetivo a realização de uma revisão sistemática de análise qualitativa dos artigos publicados em língua portuguesa, classificando-os conforme sua temática. Como resultado, descobriu-se que se tratando de uma prática esportiva, são escassos os estudos desenvolvidos no país. Neste estudo foram selecionados após uma análise crítica dezoito estudos, porém somente dois deles foram realmente voltados à área hard (campo de estudo que por sua vez engloba disciplinas como fisiologia, biomecânica, cinesiologia, anatomia, cinemática, entre outras práticas científicas). Ficou claro que o Pole Dance é uma prática de domínio feminino e que são necessários mais estudos que embasem cientificamente o desenvolvimento do Pole Dance como prática de exercício físico e modalidade esportiva.

Palavras-chave: Pole Dance; Revisão sistemática; Exercício físico; Esporte; Educação física.

\begin{abstract}
Pole Dance is a physical exercise that uses friction and opposition between the practitioner's body and a vertical bar to perform plastic, static or dynamic figures and postures, with elements of gymnastics and circus acrobatics, composing dances or sequences. acrobatics. This research on Pole Dance aimed to carry out a systematic review of qualitative analysis of articles published in Portuguese, classifying them according to their theme. As a result, it was found that in the case of a sports practice, there are few studies developed in the country. In this study, eighteen studies were selected after a critical analysis, but only two of them were really focused on the hard area (a field of study that in turn encompasses disciplines such as physiology, biomechanics, kinesiology, anatomy, kinematics, among other scientific practices). It became clear that Pole Dance is a female domain practice and that more studies are needed to scientifically support the development of Pole Dance as a practice of physical exercise and sport.
\end{abstract}

Keywords: Pole Dance; Systematic review; Physical exercise; Sport; Physical education.

\section{Resumen}

El Pole Dance es un ejercicio físico que utiliza la fricción y la oposición entre el cuerpo del practicante y una barra vertical para realizar figuras y posturas plásticas, estáticas o dinámicas, con elementos de gimnasia y acrobacias circenses, componiendo danzas o secuencias acrobáticas. Esta investigación sobre Pole Dance tuvo como objetivo realizar una revisión sistemática de análisis cualitativo de artículos publicados en portugués, clasificándolos según su temática. Como resultado se encontró que tratándose de una práctica deportiva, existen pocos estudios desarrollados en el país. En este estudio se seleccionaron dieciocho estudios luego de un análisis crítico, pero solo dos de ellos estaban realmente enfocados en el área dura (un campo de estudio que a su vez abarca disciplinas como la fisiología, la biomecánica, la kinesiología, la anatomía, la cinemática, entre otras disciplinas científicas). prácticas). Quedó claro que el Pole Dance es una práctica de dominio femenino y que se necesitan más estudios para sustentar científicamente el desarrollo del Pole Dance como práctica de ejercicio físico y deporte.

Palabras clave: Pole Dance; Revisión sistemática; Ejercicio físico; Deporte; Educación física. 


\section{Introdução}

O Pole Dance é uma atividade física que utiliza do atrito e oposição entre o corpo e uma barra vertical para a realização de figuras plásticas, estáticas ou dinâmicas, com elementos da ginástica e acrobacias circenses, compondo danças ou sequências de manobras acrobáticas.

Segundo o Sindicato dos Profissionais de Dança do Estado do Rio de Janeiro [SPDRJ] (2019), a origem do Pole Dance, para alguns estudiosos, remonta ao século XII, quando surgia na Índia o MallaKhamb, um exercício físico praticado apenas por homens e que utilizava um mastro de madeira para a realização de movimentos de força e resistência. Ainda no século XII, acredita-se ter originado o Mastro Chinês, no qual artistas circenses desenvolviam exercícios de força e acrobacias em uma barra de ferro emborrachada. A partir do século XVII, surge o Panjat Pinang, atividade de entretenimento em que os participantes escalavam árvores de Areca para alcançar presentes colocados no seu topo em casamentos e celebrações da independência da Indonésia. Todavia, a principal influência do Pole Dance em dias atuais é oriunda dos Estados Unidos da América do século XIX, onde mais especificamente as dançarinas sensuais se apresentavam próximas aos postes de sustentação de tendas circenses e posteriormente, na década de 60 do século XX, a dança realizada em postes foi inserida em clubes e boates de strip-tease, sendo seu primeiro registro datado de 1968 com a performance de Belle Jangles no clube de strip-tease Mugwump, estado do Oregon.

No Brasil, a primeira escola de Pole Dance foi fundada na cidade de Curitiba, em maio de 2008, pela professora e bailarina Grazieli Brugner, pioneira ao criar e registrar na Biblioteca Nacional do Brasil, em dezembro do mesmo ano, uma metodologia de ensino de Pole Dance para curso de capacitação de instrutores (IV Congresso Brasileiro Inspirar de Pole Dance Wellness, 2014). Sob óticas institucionais, a prática do Pole Dance no Brasil hoje é regida pela Federação Brasileira de Pole Dance [FBPOLE] (Figura 1) e a Confederação Brasileira de Aéreos e Pole Sports [CBAPS] (Figura 2).

Figura 1 Logo da FBPOLE.

\section{IFBPOLE}

Figura 2: Logo da CBAPS.

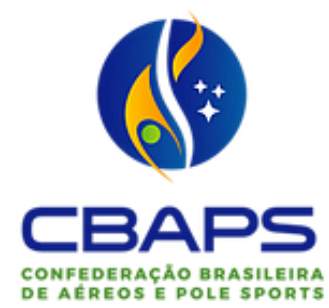

Fonte: Autores.

A FBPOLE é responsável por vários pioneirismos na área, como por exemplo, o primeiro Código de Regras e Arbitragem (já abandonado devido à filiação à POSA), realização de Pole World Cup e Pan-americanos de Pole, este último em parceria com a BSB Fitness (FBPOLE, 2021). Já a CBAPS foi fundada em Arapongas-PR, em 2019, sem fins lucrativos e visa ser reconhecida pelo Comitê Olímpico do Brasil (COB) e pela International Pole Sports Federations - IPSF (CBAPS, 2021).

Internacionalmente, o Pole Dance é reconhecido como esporte pela Pole Sports \& Arts World Federation [POSA] (Figura 3) e pela International Pole Sports Federation [IPSF] (Figura 4). A POSA é uma federação internacional criada para unir, regulamentar, organizar, liderar e desenvolver a prática e a competição do esporte e da arte do Pole Dance em todo o mundo, representando e defendendo cada uma das vinte e nove federações afiliadas, inclusive a FBPOLE (POSA, 2021). Já a IPSF é uma federação criada em 2006 por Tim Trautman e Katie Coates com o objetivo de unir os atletas, treinadores, juízes e 
entusiastas. Em 2008, após Tim entrar para a IPSF e iniciar os trabalhos com Katie, embasados em uma pesquisa de 2006 feita pela própria Katie Coates, sobre o pensamento da comunidade a respeito do Pole Sports como esporte olímpico, o termo "Pole Sports" foi criado, transformando o Pole Dance para sempre de uma atividade física realizada socialmente e para preparação física em um esporte de competição internacional. A partir de 2014, a IPSF iniciou o processo de alinhamento aos critérios da Global Association of International Sports Federation [GASF], órgão guarda-chuva composto por federações esportivas internacionais autônomas e independentes e outras organizações internacionais relacionadas a esportes e eventos (IPSF, 2015).

Figura 3: Logo da POSA.

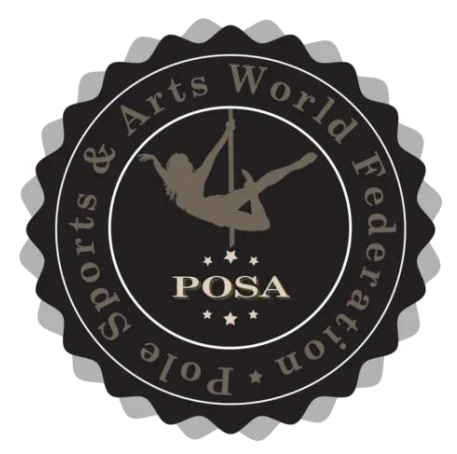

Figura 4: Logo da IPSF.

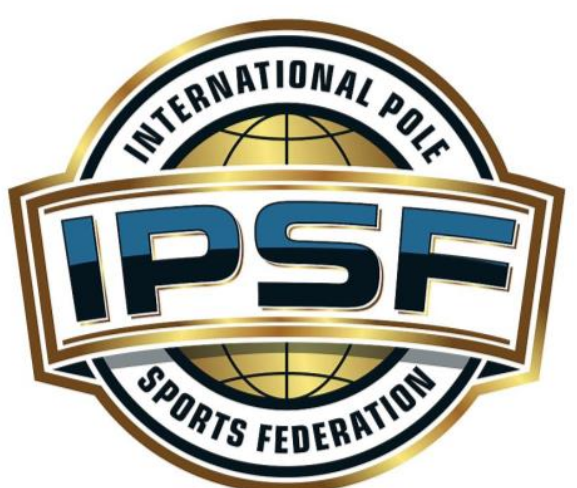

Fonte: Autores.

As competições organizadas pelo IPSF envolvem quatro modalidades de Pole Dance: Pole Sport, Ultra Pole, Pole Artístico e Para Pole. O Pole Sport envolve uma apresentação artística com ênfase no atletismo e no mérito técnico. Ultra Pole é uma modalidade que visa estimular a criatividade e a criação de novos movimentos e é realizada por meio de apresentações em formato de batalha em que os competidores expressam suas habilidades acrobáticas e inovadoras. Por sua vez, o Pole Artístico tem como objetivo estimular a criatividade e a liberdade de expressão, enfatizando a interpretação artística. Por fim, tem-se o Para Pole em que os atletas competem em classes esportivas definidas de acordo com a sua deficiência (IPSF, 2015).

Há ainda diversos outros campeonatos de nível nacional e regional, que envolvem as modalidades de Pole Sport, Pole Artístico e/ou Exotic Pole. Os campeonatos de Pole Sport seguem o código de pontos da IPSF ou da POSA, tendo o competidor que executar os movimentos listados e apresentados previamente à organização (similarmente ao que é visto nas competições de ginástica artística, patinação artística e salto ornamental). Já em relação ao Pole Artístico, tal categoria não segue os referidos códigos de pontos, tendo cada competição um regulamento próprio. Por fim, ainda existem campeonatos de Exotic Pole, categoria essa que também não dispõe de código de pontos e cuja modalidade se diferencia por utilizar movimentos de alta complexidade, com muito floorwork (movimentos de chão), movimentos de pernas e handstands (parada de mão) e poucos movimentos na barra.

Popularmente, o Pole Dance possui várias vertentes. Desde já é importante citar que por conta de diversas questões de cunho regional, cultural e principalmente mercadológico, não é incomum encontrar variações de nomenclaturas envolvendo o Pole Dance, tais como: Pole Dance Glamour, Pole Dance Contemporâneo, Pole Dance Heels, dentre outros. De fato, tal diversidade pode causar alguma estranheza ou confusão, contudo algumas vertentes são mais utilizadas, conhecidas e mesmo estabelecidas na sociedade hodierna. O Pole Sensual é a vertente mais conhecida por se aproximar dos espetáculos de strip clubs e ter significativo foco na sensualidade ao explorar o contato visual, as expressões faciais, o floorwork e a vestimenta. O Exotic Pole está relacionado aos pole dancers russos e, como já mencionado, utiliza de movimentos de alta complexidade, 
misturando floorwork e handstands em coreografias que combinam ritmo, técnica, fluidez, força e flexibilidade. Merece destaque ainda o Pole Acrobático ou Pole Fitness, que é considerado a base de aprendizagem para qualquer outra modalidade e que possui como objetivo o ensino das técnicas para execução de giros, transições, inversões, figuras, quedas, os quais poderão ser executados isoladamente ou combinados. Ainda se faz importante destacar que em muitos casos, há um terreno convergente e mútuo entre tais vertentes, que de certa forma pode dificultar as delimitações, não sendo raro observar coreografias que comecem em uma vertente e transitem por outras. Isso demonstra que o Pole Dance tanto como exercício físico padronizado ou mesmo como modalidade desportiva competitiva carece de uma maior definição e organização para com suas múltiplas possíveis ramificações, já que se faz uma prática ainda incipiente em âmbito organizacional.

Tendo em vista que as práticas de atividade física, exercícios físicos e modalidades desportivas são objetos de pesquisa do campo da Educação Física e ainda configurando por sua vez o Pole Dance como uma dessas práticas esportivas incipientes, já que se encontra institucionalizada e envolve esforço físico vigoroso e o uso de habilidades motoras complexas por parte de seus praticantes (Barbanti, 2006), faz-se necessário o desenvolvimento de novas pesquisas sobre a temática. Ademais, sendo o esporte recente no cenário brasileiro, ainda são escassos os estudos desenvolvidos no país. Dessa forma, o objetivo geral deste estudo é classificar os artigos garimpados de acordo com a sua temática, mais especificamente suas áreas de estudo. Mais especificamente, objetiva-se ainda identificar o quão a Educação Física acolheu o Pole Dance no âmbito da pesquisa.

Portanto, este trabalho se torna relevante, pois tende a mediar futuras pesquisas, mapeando, direcionando e orientando profissionais de diferentes áreas na busca da atualização e construção de novas diretrizes, contribuindo para enriquecimento da literatura acerca de variados temas relacionados ao Pole Dance, reafirmando cada vez mais a emergência dessa prática como esporte e assim integrante do campo da Educação Física.

\section{Metodologia}

Neste estudo foi realizada uma revisão sistemática de análise qualitativa (Gomes \& Caminha, 2014) acerca dos estudos encontrados em língua portuguesa com o objetivo de classificá-los de acordo com a sua temática. Segundo Sampaio e Mancini (2007), a revisão sistemática é o método que utiliza como fonte outros estudos já existentes na literatura sobre determinado tema, a partir dos quais é realizado um resumo contendo as principais informações apresentadas e que permite uma maior visão sobre as evidências já apuradas, facilitando, assim, a comparação de resultados e a identificação de questões que ainda precisam ser estudadas, auxiliando futuras investigações. Este método de pesquisa abrange: definição de uma pergunta científica; identificação da base de dados e de estratégias de busca, guiada por palavras-chave; estabelecimento de critérios para a seleção dos artigos e condução da busca, comparando as buscas dos examinadores; aplicação dos critérios na seleção dos artigos; justificativa das possíveis exclusões; análise crítica e avaliação de todos os estudos incluídos; preparo de um resumo crítico; e por fim, apresentação de uma conclusão, informando a evidência sobre os efeitos da intervenção.

Como primeiro passo para a elaboração da presente revisão sistemática, restou definida a necessidade de responder ao seguinte questionamento: quais as temáticas abordadas nos estudos brasileiros envolvendo o Pole Dance? Em seguida foi definida a plataforma Google Acadêmico como a base de dados para a busca de quaisquer tipos de estudos relacionados ao tema em discussão. A busca foi concretizada na data de 22 de fevereiro de 2021, utilizando-se as seguintes palavras-chave: “pole dance", "poledance", "Pole Dance” e "pole-dance”, sendo que a busca que gerou maior resultado foi com a utilização da palavra chave "pole dance", razão pela qual foi escolhida como critério de seleção dos estudos. Como limitadores da pesquisa, definiu-se que o termo "pole dance" deveria constar no título da publicação e os textos deveriam estar em sua íntegra e na língua portuguesa. Com a busca, foram obtidos vinte e nove resultados.

Por fim, foi realizada a leitura completa de todos os textos e a análise do seu conteúdo (Bardin, 2004), tendo eles sido 
classificados em: a) elegíveis: estudos obtidos em sua integralidade e que abordam questões específicas relacionadas ao Pole Dance; b) não elegíveis: estudos duplicados (aqueles que já haviam sido selecionados anteriormente) e incompletos (aqueles que não puderam ser obtidos na íntegra), sem possibilidade de inclusão no presente estudo. Ao final, dezoito estudos foram elegíveis para as discussões.

\section{Resultados e Discussão}

Após a leitura e análise de conteúdos, segundo as diretrizes de Bardin (2004), os textos foram divididos em dez categorias dispostas em ordem alfabética, conforme a Figura 5.

Figura 5 - Categorização do conteúdo.

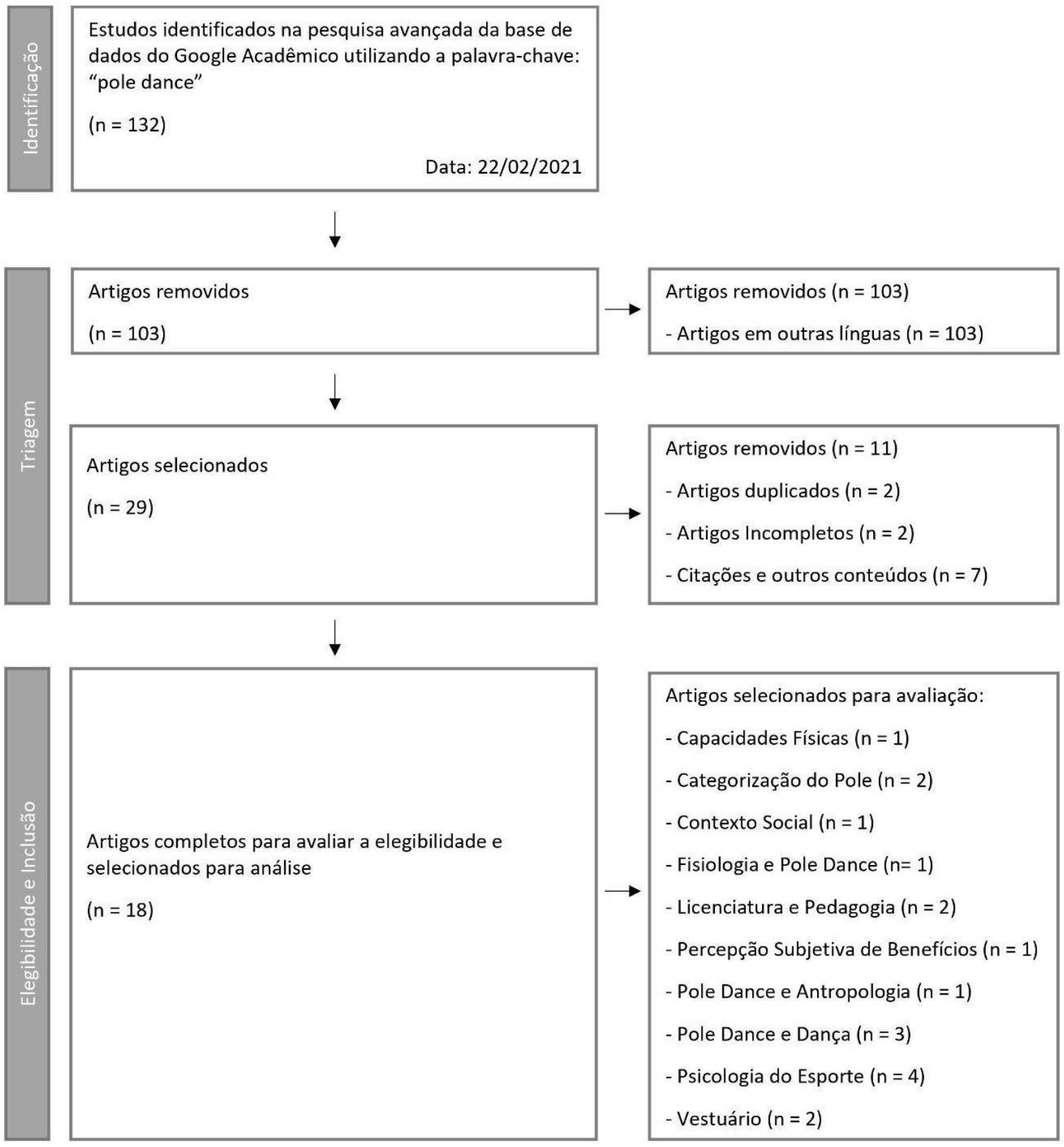

Fonte: Autores. 
$\mathrm{O}$ artigo "Comparação da força, flexibilidade e resistência de mulheres praticantes de treinamento de força e praticantes de Pole Dance" (Rosin et al., 2017), foi categorizado na linha de Capacidades Físicas devido ao fato de que seu objetivo principal foi comparar as capacidades físicas entre mulheres praticantes de treinamento de força e praticantes de Pole Dance. $\mathrm{O}$ estudo foi feito com vinte e duas mulheres entre dezoito e trinta anos de idade sendo onze pertencentes ao grupo de praticantes de treinamento de força e onze pertencentes ao grupo de praticantes de Pole Dance, chegando a conclusão de que mulheres praticantes de treinamento de força possuem maior força e menor flexibilidade, principalmente dos membros inferiores, quando comparadas às praticantes de Pole Dance, não apresentando diferenças significativas das capacidades físicas analisadas em relação aos outros grupamentos musculares (músculos dos membros superiores e músculos do abdome).

Já os trabalhos de conclusão de curso (TCC) "Pole dance: dança ou esporte?" (Santos, 2018) e "Pole Dance: considerações sobre a prática e sua multiplicidade" (Cury, 2018) foram colocados na classificação de Categorização do Pole Dance, uma vez que seus objetivos eram discorrer sobre em qual contexto o Pole Dance mais se encaixa, no dos esportes, da dança sensual ou do lazer, ambos usando de pesquisa exploratória em suas metodologias. O primeiro defende a ideia de que seria de suma importância a presença de profissionais de Educação Física na realização e/ou na supervisão de sua prática, uma vez que o Pole Dance como exercício físico tanto agrega e molda capacidades físicas, metabólicas e estimula a promoção de saúde, como pode oferecer risco à integridade física dos praticantes. Já o segundo defende o Pole Dance como uma prática híbrida, não diferenciando tal prática entre dança, exercício físico, esporte, dentre outras rotulações, já que o Pole Dance pode trabalhar com públicos diversos (crianças, adolescentes e adultos). Ainda é destacado no texto que o Pole Dance hoje vem sendo utilizado como difusor de aspectos que envolvem a autoestima feminina: sensualidade, feminilidade, empoderamento, além de ser um composto multidimensional, não devendo suas nuances serem excludentes, mas sim abrangentes. Tal classificação abre as portas para a seguinte categoria.

A "Mulher Escarlate: uma exposição sobre empoderamento da mulher em espaços urbanos através do Pole Dance" (Achôa, 2019) compreendido na categoria Contexto Social, desenvolveu uma exposição multimídia que retrata mulheres expressando-se em movimentos do Pole Dance. Registrando mulheres de diferentes corpos e idades executando a sua compreensão do Pole Dance; assim como retratou a diversidade do Pole Dance por meio de documentação visual dessas mulheres performando a vertente com que mais se identificavam. E desdobrou uma linguagem/identidade visual que melhor conversou com o público alvo, também organizou um percurso expográfico para o melhor entendimento da lógica do trabalho. Concluindo que o projeto cumpriu com os objetivos estabelecidos, tanto o geral quanto os específicos. O percurso expográfico deste trabalho foi organizado de forma linear de modo que também estabeleceu uma crescente familiaridade do público com as modelos e a realidade das dançarinas. O tema se mostrou cada vez mais pertinente no decorrer do desenvolvimento deste trabalho, desde a leitura de artigos relacionados, aproximação com o público alvo e de forma mais pungente na fase da captura das mídias. A exposição foi idealizada para atender as mais diversas finalidades - seja curiosidade, interesse, admiração, busca do conhecimento, entre outras - dentro de um espaço neutro que retrata, com naturalidade e organicidade, a beleza do Pole Dance além do teor erótico e sexual.

A monografia do Curso de Especialização em Treinamento de Força e Hipertrofia "Respostas fisiológicas induzidas pela prática de Pole Dance" (Silva, 2017) foi classificada como Fisiologia e Pole Dance, seguindo a mesma linha de pensamento da categoria Capacidades Físicas, uma vez que seu foco principal foi o estudo do comportamento de variáveis fisiológicas induzido pela prática do Pole Dance. O estudo foi realizado com seis mulheres, de idade compreendida entre trinta e sessenta e dois anos e foi verificado que, de acordo com as seguintes médias de idade, percentual de gordura e massa magra (aproximadamente trinta anos de idade, $25 \%$ e $45 \%$, respectivamente), o comportamento apresentou as seguintes características: frequência cardíaca atingindo seu pico na segunda sequência de exercício, 134.1 bpm, estabilizando-se ao final do teste com 115.2 bpm; média de gasto energético de 379,11 kcal de uma sequência de exercício para outra (sendo o pico de 
429 na quarta e última sequência de exercício); a média de lactato foi de 3,62mM no início, 5,25mM logo após os exercícios e 4,48mM após a recuperação (10min); no teste de PSE (percepção subjetiva de esforço) as avaliadas sentiram maior exigência de força no ombro (único que obteve acima de 50\%).

Os trabalhos "O ensino do Pole Dance na escola: desafios e possibilidades" (Rizzo et al.,2019) e "Pedagogia Desportiva e o ensino da Dança do Varão na escola: uma pesquisa-ação sobre os impactos nas aulas de Educação Física" (Rizzo et al., 2020) foram incluídos na categoria Licenciatura e Pedagogia. Ambos apresentam como objetivo desenvolver e propor um modelo pedagógico de ensino do Pole Dance nas aulas de Educação Física escolar com base na teoria interacionista, uma vez que não existem quaisquer modelos vigentes na atualidade. A proposta tem como fundamento o fato do Pole Dance ser uma prática prazerosa e eficaz, favorecendo a origem de relações interpessoais e proporcionando uma prática corporal não convencional ao ambiente escolar. Para o desenvolvimento dos trabalhos, foi realizada uma pesquisa-ação qualitativa, dividida em três etapas: 1) questionário destinado aos alunos para compreender quais conhecimentos já possuíam sobre Pole Dance; 2) organização de encontros baseados nas três dimensões do conteúdo, quais sejam conceitual, procedimental e atitudinal, sendo que na prática dos movimentos característicos do Pole Dance foi desenvolvida uma implementação de ensino sustentada pela "teoria interacionista"; 3) questionário aplicado aos envolvidos para analisar alterações ou não na percepção dos alunos em relação à prática de Pole Dance. Ao final, os estudos concluíram terem alcançado seu objetivo ao apresentar uma possibilidade pedagógica para o ensino e aprendizagem do Pole Dance nas aulas de Educação Física, em prática condizente com a realidade da escola.

Na categoria Percepção Subjetiva de Benefícios foi incluído um estudo transversal de caráter exploratório: "Percepção dos benefícios da prática da modalidade de Pole Dance Fitness" (Oliveira, 2016). A amostra foi composta por vinte alunas de uma academia de Pole Fitness com idade entre quinze e cinquenta anos e que praticavam a atividade há pelo menos seis meses. Quanto à metodologia, foi aplicado um questionário cujo principal objetivo foi verificar a percepção de alunas praticantes de Pole Dance sobre os benefícios dessa modalidade, por ser o Pole Dance Fitness uma modalidade nova e que vem despertando interesse em novos praticantes. Como resultado, foi percebido que a maioria das mulheres procurava o Pole Dance Fitness para praticar alguma atividade física, verificando-se também a percepção de melhora em relação à saúde, onde $95 \%$ das mulheres participantes relataram perceber melhora em suas estruturas físicas e seus condicionamentos.

Na categoria Pole Dance e Antropologia o trabalho "Viva o matriarcado Pole Dance - uma etnografia das relações entre corpo, gênero e cidade na prática do Pole Dance" (Gonçalves, 2017) mapeou seis mulheres em um único estúdio de Pole Dance na cidade de Niterói/RJ. Após isso, foram realizadas conversas e não entrevistas, para que houvesse diálogos menos mecanizados e com maior abertura. Seguindo a mesma lógica, foram escolhidas pessoas com quem a autora tinha um mínimo de proximidade. A proposta foi, a partir de um trabalho de campo, perceber como essas vidas se cruzam e como se alteram mutuamente a partir do contato com a prática e observar de que forma a cidade se insere nesse processo e entender qual a relação entre o Pole Dance e o feminismo. De acordo com a autora, foi visto que a prática do Pole Dance tratou de feridas tão profundas e dolorosas que pareciam incuráveis, descobrindo formas de mulheres se empoderarem.

Os trabalhos "Pole Dance: contextos e aproximações com estudos de Rudolf Laban" (Oliveira, 2016), "Pole Dance: Estudo de Laban aplicados à verticalidade do Pole" (Brito, 2018) e "A experiência do corpo no Pole Dance: por uma dimensão carnal do conhecimento" (Pereira, 2015) estão abrangidos na categoria Pole Dance e Dança. No primeiro, Oliveira (2016) realizou uma seleção de textos sobre Laban, para embasar neles a aproximação do Pole Dance e da Eukinética. Objetivou-se assim contribuir com a vivência e o ensino do Pole Dance a partir do conhecimento da qualidade e das características de seus movimentos básicos, que podem ser explorados, atualizados e reconfigurados em novas construções coreográficas futuras pelo professor e pelo praticante. Para a autora, a discussão apresentada traz possibilidades de ampliação futura no âmbito da dança, ou seja, trabalhar o Pole Dance como processo criativo e ainda a possibilidade de investigar como acontece o ensino do Pole 
Dance em estúdios. No segundo trabalho, Brito (2018) realizou uma pesquisa de campo descritiva e um estudo de caso. Realizaram-se experimentos de trechos coreográficos de dança para o Pole Dance com o interesse de associar a esta técnica os fatores de Laban estudados e experimentados em cada ponto de processo de movimentação coreográfica. O estudo levantou dados e objetivou sincronicamente descrever os pontos potencializadores e bloqueadores encontrados no decorrer dos experimentos e laboratórios de criação desta pesquisa. A partir dos experimentos e análises foi possível reconhecer as relações entre o Pole Dance e os conhecimentos produzidos na área da dança ou mesmo de reconhecer o Pole Dance como um gênero de dança, porém isto não invalida a possibilidade de trabalhar o Pole Dance também como exercício físico esportivo, isolando seus movimentos direcionados a modificação corporal. Ao fim desta categoria, Pereira (2015) traz uma pesquisa qualitativa que foi dividida em dois momentos específicos. Primeiramente, uma análise do discurso obtido em entrevista contendo diversos temas relacionados ao Pole Dance, na qual se analisou a fala de bailarinos de Pole Dance que tinham uma prática sistemática há no mínimo dois anos e já tinham participado de no mínimo uma competição. Posteriormente, foram analisados vídeos coletados da internet de dois campeonatos diferentes que ocorreram no ano de 2015. A partir dos dados obtidos, foram realizadas discussões sobre: 1) contextualização histórica do Pole Dance e seu diálogo com outras práticas e discussão sobre seus tabus, 2) elementos técnicos e estéticos que compõem a prática e, 3) as sensações do corpo em contato com a prática e os seus significados para os praticantes. Dessa forma, o trabalho visou analisar a experiência do corpo no Pole Dance e como a mídia interfere nas competições e apresentações de Pole Dance; além de desmistificar o tabu do Pole Dance como uma prática ligada apenas ao sensual (sexual) e entender o que diz o corpo que dança Pole Dance.

Outra categoria, denominada Psicologia do Esporte, abrangeu quatro estudos que abordam tópicos como a motivação para a procura e permanência de mulheres nas aulas de Pole Dance e também o reflexo da prática à autoimagem das participantes. O primeiro trabalho, "Motivação para prática do pole dance como atividade física por mulheres" (Lopes et al., 2019), utilizou de uma pesquisa de natureza quantitativa e descritiva, na qual aplicou às participantes um questionário socioeconômico e um questionário Exercise Motivations Inventory-2 (Questionário de Motivação para o Exercício) composto por cinquenta e um itens. Como resultado observou que o maior fator motivacional que leva as mulheres entrevistadas à prática do Pole Dance é a melhora/manutenção da saúde e o prazer que o exercício da modalidade pode oferecer. No artigo intitulado “Autoimagem e autoestima de mulheres praticantes de pole dance” (Godinho, 2018) foi realizada uma pesquisa de natureza aplicada e descritiva, tendo sido utilizadas a Escala de Silhueta e a Escala de Autoestima para verificar a imagem real e a ideal da silhueta de cada participante e os sentimentos de respeito e de aceitação de si mesmo. Os dados foram analisados por meio de estatística descritiva e de frequência simples e concluiu que a maioria das praticantes se encontra insatisfeita com a imagem corporal, porém apresenta autoestima boa ou elevada, fator este influenciado pela prática regular de Pole Dance. Já o trabalho intitulado de "Motivo na aderência de mulheres a prática regular de aulas de Pole Dance na cidade de Criciúma/SC" (Fernandes, 2012) e apresentado ao curso de Educação Física, por meio de um estudo de campo de natureza qualitativa e descritiva, realizou uma entrevista semiestruturada com as participantes questionando os motivos que levaram à procura do Pole Dance, os objetivos pretendidos antes e depois de iniciarem a prática de Pole Dance e os motivos que levam as participantes a permanecerem praticando a modalidade. Os resultados apontam que a busca pela modalidade ocorreu principalmente em razão da indicação de terceiros e a curiosidade e que os objetivos iniciais ainda permanecem, porém acrescidos de novos, os quais juntamente com as mudanças provocadas na vida pessoal das participantes (melhora da autoestima, motivação para outros exercícios, estética, postura, confiança e feminilidade) influenciam para que elas permaneçam praticando o Pole Dance. Finalmente, a dissertação de mestrado em Psicologia, nomeada de "Redescobrindo sersi-mesmo: a existencialidade de mulheres praticantes de Pole Dance" (Ferreira, 2015), realizou uma pesquisa qualitativa e utilizou dos pressupostos da Psicologia Fenomenológica de Merleau-Ponty para a análise dos dados obtidos. Participaram do estudo doze mulheres praticantes de Pole Dance a mais de três meses e residentes na cidade de Manaus/AM. As participantes 
foram entrevistadas e a partir de seus discursos foram criadas categorias temáticas: Começando a "polear"; Obstáculos versus Superação; Pré-Conceitos; Re-descobrindo ser-si-mesmo; e "O que significa pole dance para você?”. Ao final, analisando as respostas apresentadas, o trabalho concluiu que mesmo diante das diferentes experiências sobre a mesma prática (fatores motivacionais que levaram as praticantes a iniciarem e a permanecerem na atividade, as mudanças na autoestima, os preconceitos enfrentados e as amizades estabelecidas), o Pole Dance permitiu às participantes se re-descobrirem, transformando a forma como elas se veem e percebem o seu corpo na interação com-si-mesmas e com-o-mundo.

Por fim, dois trabalhos foram incluídos na categoria Vestuário, "Estudo ergonômico no desenvolvimento de vestuário adequado para prática de Pole Dance" (Cilense et al., 2017) e "Vestuário ergonômico para a prática de Pole Dance: o conforto térmico como requisito projetual". O primeiro realizou uma pesquisa de campo com quatro professores e seis alunas de Pole Dance e associou a ergonomia para a construção do questionário sendo que, após sua aplicação, foi feita a indicação do vinil como o tecido de maior similaridade à pele com relação à aderência e a indicação de que tops devam promover a compressão sem bojos e sem ter comprimento alongado. Já o segundo artigo contou com cinquenta e duas mulheres, com idades entre dezoito e quarenta anos e concluiu que devido à sudação e ao cansaço muscular, há a necessidade de tecidos tecnológicos que visem maior eficiência em troca de calor do corpo com o ambiente, por conta da relação do atrito do corpo com a barra, tecidos com toques de emborrachado também são de suma importância, além de que um estudo das tarefas realizadas deve ser mais bem estruturado quanto ao processo de desenho da roupagem e as formas anatômicas, visando a melhor adaptação ao corpo e facilitação das atividades dos usuários.

A revisão realizada deixa evidente que embora a prática do Pole Dance não seja recente, poucos são os estudos envolvendo a modalidade. Todas as publicações encontradas são posteriores ao ano de 2016, com exceção do trabalho de Fernandes (2012). Embora em crescimento, constata-se que ainda há uma carência de pesquisa sobre o assunto principalmente no campo da Educação Física. O Pole Dance já é reconhecido como uma prática esportiva e não foram encontradas pesquisas que envolvam treinamento e biomecânica dos movimentos, mas tão somente um único trabalho que aborda as capacidades físicas e outro que aborda os aspectos fisiológicos que envolvem a prática. Em contrapartida, é interessante destacar que as pesquisas encontradas envolvem diversas áreas do conhecimento como design, moda, dança, psicologia, antropologia e ciências sociais, o que demonstra a complexidade e ampla capacidade de exploração mercadológica que envolve o Pole Dance.

Destaca-se que muitos estudos estão relacionados aos aspectos subjetivos do Pole Dance envolvendo questões que abordam a motivação para a busca e permanência nas aulas, os reflexos na autoimagem e a percepção sobre os benefícios e alterações provocados pela prática. Também, tem-se que a maioria dos estudos foi realizada como Trabalhos de Conclusão de Curso de Graduação, perfazendo treze publicações, além de uma monografia de curso de especialização e uma dissertação de mestrado, contra tão somente seis artigos, evidenciando mais uma vez a escassez de pesquisas científicas sobre a temática.

Com relação ao público, as pesquisas sugerem a realidade da maioria dos estúdios de Pole Dance no Brasil, ou seja, que a grande maioria dos praticantes de Pole Dance são mulheres em idade adulta. Ainda nos dias atuais, o Pole Dance carrega uma imagem estereotipada de que a prática envolve tão somente uma dança sensual em um mastro vertical e assim uma crença de que a modalidade é destinada principalmente às mulheres. No entanto, vários são os contextos em que o Pole Dance se encaixa, podendo ser explorado como dança, esporte ou até mesmo uma atividade física para promoção de saúde e bem-estar e por isso é capaz de abranger diferentes públicos, inclusive o masculino e o infantil, ainda pequenos e incipientes na realidade brasileira.

\section{Conclusão}

Portanto, o que se verifica é que o Pole Dance é uma modalidade crescente no país e no mundo e que precisa ser mais explorada cientificamente pelas diversas áreas do conhecimento, em especial pela área da Educação Física tendo em vista o 
recente reconhecimento do Pole Dance como esporte. Não obstante, a exploração do Pole Dance em suas diversas possibilidades - dança, esporte ou atividade física - pela área científica poderá contribuir para a difusão dos benefícios proporcionados por sua prática e para a superação dos diversos preconceitos que envolvem a modalidade. Recomendam-se mais estudos sobre o Pole Dance, principalmente em campos da chamada área hard, que por sua vez engloba a fisiologia, biomecânica, cinesiologia, anatomia, cinemática, entre outras práticas científicas. Assim, o Pole Dance poderia ter maiores alicerces e suportes científicos para seu desenvolvimento geral, incluindo sua vertente desportiva de alto-rendimento no campo da Educação Física.

\section{Referências}

Achôa, J. F. (2019). A mulher escarlate: uma exposição sobre empoderamento da mulher em espaços urbanos através do pole dance. Trabalho de Conclusão de Curso (Graduação), Universidade Federal de Santa Catarina, Florianópolis, SC, Brasil. Recuperado 22 de fevereiro, 2021 , de https://repositorio.ufsc.br/handle/123456789/202421

Almeida, V. L. T. (20016). Percepção dos benefícios da prática da modalidade pole dance fitness. Trabalho de Conclusão de Curso, Centro Universitário de Brasília, Brasília, DF, Brasil. de https://repositorio.uniceub.br/jspui/handle/235/10653

Baldin, A. E. C., \& Menegucci, F. (2017). Vestuário ergonômico para a prática de pole dance: o conforto térmico como requisito projetual. Projética, 8(2). http://www.uel.br/revistas/uel/index.php/projetica/article/view/27330

Barbanti, V. J. (2006) O que é esporte?. Revista Brasileira de Atividade Física e Saúde, (2). 54-58. http://sistemas.eeferp.usp.br/myron/arquivos/7844237/e169c31d328f4fa63211594b6cbf6075.pdf

Brito, I. S. (2018). Pole dance: estudos de Laban aplicados à verticalidade do pole. Trabalho de Conclusão de Curso, Universidade do Estado do Amazonas, Manaus, AM, Brasil. http://repositorioinstitucional.uea.edu.br/handle/riuea/1640

Cilense, I. T. M., \& Silva, M. A. R. (2019). Estudo ergonômico no desenvolvimento de vestuário adequado para prática de pole dance. In: L. S. Bertoso (Org.) Na estante da moda 2. Ponta Grossa: Atena, 2019. https://www.atenaeditora.com.br/wp-content/uploads/2019/05/e-book-Na-Estande-da-Moda2.pdf.pdf.

Confederação Brasileira de Aéreos e Pole Sports. Sobre nós. (2021). https://www.cbaps.org/copia-filiacao

Cury, C. N. (2018). Pole dance: considerações sobre a prática e sua multiplicidade. Trabalho de Conclusão de Curso, Universidade Federal do Rio Grande do Sul, Porto Alegre, RS, Brasil. https://www.lume.ufrgs.br/handle/10183/217421

Federação Brasileira De Pole Dance. Quem somos. (2021). https://www.fbpole.org.br/federacao-brasileira-pole-dance/fbpole

Fernandes, J. M. (2012). Motivo na aderência de mulheres à prática regular de aulas de pole dance na cidade de Criciúma-SC. Trabalho de Conclusão de Curso, Universidade do Extremo Sul Catarinense, Criciúma, SC, Brasil. http://repositorio.unesc.net/handle/1/1476

Ferreira, C. F. (2015). Redescobrindo ser-si-mesmo: a existencialidade de mulheres praticantes de pole dance. Dissertação de mestrado, Universidade Federal do Amazonas, Manaus, AM, Brasil. https://tede.ufam.edu.br/handle/tede/4892

Godinho, A. P. (2018). Auto imagem e autoestima de mulheres praticantes de pole dance. Trabalho de Conclusão de Curso, Universidade do Sul de Santa Catarina, Palhoça, SC, Brasil. https://www.riuni.unisul.br/handle/12345/7314

Gomes, I. S., \& caminha, I. O. (2014). Guia para estudos de revisão sistemática: uma opção metodológica para as Ciências do Movimento Humano. Movimento, v.20, n.1, pp.395-411, jan/mar de 2014. https://seer.ufrgs.br/Movimento/article/view/41542/28358

Gonçalves, A. C. (2017). "Viva o matriarcado pole dance" - uma etnografia das relações entre corpo, gênero e cidade na prática do pole dance. Trabalho de Conclusão de Curso, Universidade Federal Fluminense, Niterói, RJ, Brasil. Recuperado em 22 de fevereiro, 2021, de https://app.uff.br/riuff/handle/1/6873

International Pole Sports Federations. Disciplines of Pole. http://www.polesports.org/world-pole/pole-disciplines/

IV Congresso Brasileiro Inspirar De Pole Dance Wellness. http://faculdadeinspirar.com.br/poledance/o-congresso/grazzy-brugner

Mattes, V., Lopes, E. R., \& Medeiros, T. E. (2018). Motivação para prática do Pole Dance como atividade física por mulheres. Saúde em Revista, 18(49), https://www.metodista.br/revistas/revistas-unimep/index.php/sr/article/view/3795

Oliveira, A. K. S. (2016). Pole dance: contextos e aproximações com os estudos de Rudolf Laban. Trabalho de Conclusão de Curso, Universidade Federal do Rio Grande do Norte, Natal, RN, Brasil. https://monografias.ufrn.br/jspui/handle/123456789/2943

Pereira, T. C. F. (2015). A experiência do corpo no pole dance: por uma dimensão carnal do conhecimento. Trabalho de Conclusão de Curso, Universidade Federal de São Paulo, Santos, SP, Brasil. de https://repositorio.unifesp.br/handle/11600/53275

Pole Sports \& Arts World Federation. The Federation. https://www.posaworld.org/the-federation/

Rizzo, D. T. S., Melo, R. Z., Sousa, A. P. M., \& Golin, C. H. (2019). O ensino do Pole dance na escola: desafios e possibilidades. Revista FSA, 16(6). http://www4.unifsa.com.br/revista/index.php/fsa/article/view/1875 
Rizzo, D. T. S., Sousa, A. P. M., Golin, C. H., \& Aranha, A. C. M. (2020). Pedagogia desportiva e o ensino da dança do varão na escola: uma pesquisa-ação sobre os impactos nas aulas de educação física. Revista Brasileira de Educação, Tecnologia e Sociedade, 13(1). http://www.brajets.com/index.php/brajets/article/view/689

Rosin, R., Bortoluzzi, R., Roncada, C., \& Tiggemann, C. L. (2017). Comparação da força, flexibilidade e resistência de mulheres praticantes de treinamento de força e praticantes de pole dance. Revista brasileira Ciência e Movimento, 25(3). https://portalrevistas.ucb.br/index.php/RBCM/article/view/18/pdf

Sampaio, R.F., \& Mancini, M.C. (2007). Estudos de revisão sistemática: um guia para síntese criteriosa da evidência científica. Revista Brasileira de Fisioterapia. São Carlos, 11(1), 83-89. http://www.scielo.br/scielo.php?script=sci_arttext\&pid=S1413-35552007000100013\&lng=en\&nrm=iso

Santos, R. O. (2018). Pole dance: dança ou esporte?. Trabalho de Conclusão de Curso, Universidade Federal do Rio Grande do Norte, Natal, RN, Brasil. https://monografias.ufrn.br/jspui/handle/123456789/8665

Silva, J. M. (2017). Respostas fisiológicas induzidas pela prática de pole dance. Trabalo de Conclusão de Curso de Especialização, Universidade Federal do Paraná, Curitiba, PR, Brasil. de https://www.acervodigital.ufpr.br/handle/1884/54704.

Sindicato Dos Profissionais De Dança Do Estado Do Rio De Janeiro. Apostila de conteúdo e referência para a prova teórica de Pole Dance. https://spdrj.com.br/wp-content/uploads/sites/150/2019/09/APOSTILA-DE-POLE-DANCE.pdf 